\title{
COMPRESSIBLE TWO-PHASE FLOWS BY CENTRAL AND UPWIND SCHEMES
}

\author{
Smadar Karni $^{1}$, Eduard KirR ${ }^{2}$, Alexander Kurganov ${ }^{3}$ and Guergana Petrova ${ }^{4}$
}

\begin{abstract}
This paper concerns numerical methods for two-phase flows. The governing equations are the compressible 2-velocity, 2-pressure flow model. Pressure and velocity relaxation are included as source terms. Results obtained by a Godunov-type central scheme and a Roe-type upwind scheme are presented. Issues of preservation of pressure equilibrium, and positivity of the partial densities are addressed.
\end{abstract}

Mathematics Subject Classification. 35L65, 65M06, 76N15, 76 T99.

Received: December 28, 2002. Revised: February 24, 2004.

\section{INTRODUCTION}

Understanding the dynamics of fluids consisting of several fluid components is of great interest in a wide range of physical flows. Examples extend from studying the dynamics and stability of propagating interfaces, to studying the behavior of droplet suspensions and bubbly flows.

Multicomponent flows consist of several fluids separated by material interfaces, each occupying its own space. The governing equations are the equations of motion of the individual species, supplemented by boundary conditions at material interfaces. Further simplifications may be introduced. Assuming that all fluid components may be described by a single pressure and single velocity function, and summing over the species momentum and energy equations, a reduced model may be derived for the total momentum and energy of the fluid mixture. This model may serve as basis for computations of multicomponent flow problems, provided the number of interfaces is not too large. Recent years have witnessed a growing interest in developing numerical methods for such models and produced a number of successful methods, where the key feature is a built-in ability to recognize and respect pressure equilibrium between different fluid components (for an overview, see for example [1] and the references therein).

In bubbly flows or liquid suspensions, the number of interfaces is too large to track, and it is widely accepted that average flow characteristics are sufficient to describe the relevant macroscopic dynamics. Averaging the equations, for example across the channel in Figure 1.1, yields models in which at every point in space, all fluid

\footnotetext{
Keywords and phrases. Euler equations, two-phase flows, numerical methods, central schemes, upwind schemes.

1 Department of Mathematics, University of Michigan, Ann Arbor, MI 48109, USA, and Department of Computer Science and Applied Mathematics, The Weizmann Institute of Science, Rehovot 76100, Israel. e-mail: karni@math.lsa.umich.edu

2 Department of Mathematics, University of Chicago, Chicago, IL 60637, USA. e-mail: ekirr@math.uchicago.edu

3 Department of Mathematics, Tulane University, New Orleans, LA 70118, USA. e-mail: kurganov@math.tulane.edu

4 Department of Mathematics, Texas A\&M University, College Station, TX 77843, USA. e-mail: gpetrova@math.tamu.edu
} 
components co-exist with certain volume fractions (see for example [4,21]). Such multiphase models are inherently nonconservative due to momentum and energy exchange terms between the phases. They require closure relations which are not available from first physical principles, and even when motivated by physical considerations yield controversial results [4,21]. Pressure differences may develop between the phases, but they cannot be sustained and tend to equilibrate. Yet assuming a single (equilibrium) pressure leads to ill-posedness due to loss of time-hyperbolicity of the governing equations [21]. This ill-posedness is not only an intriguing outcome, but also a major obstacle in the design of numerical methods. Hyperbolicity is also lost in two-pressure models if one of the phases is assumed incompressible [14,15,19,22].
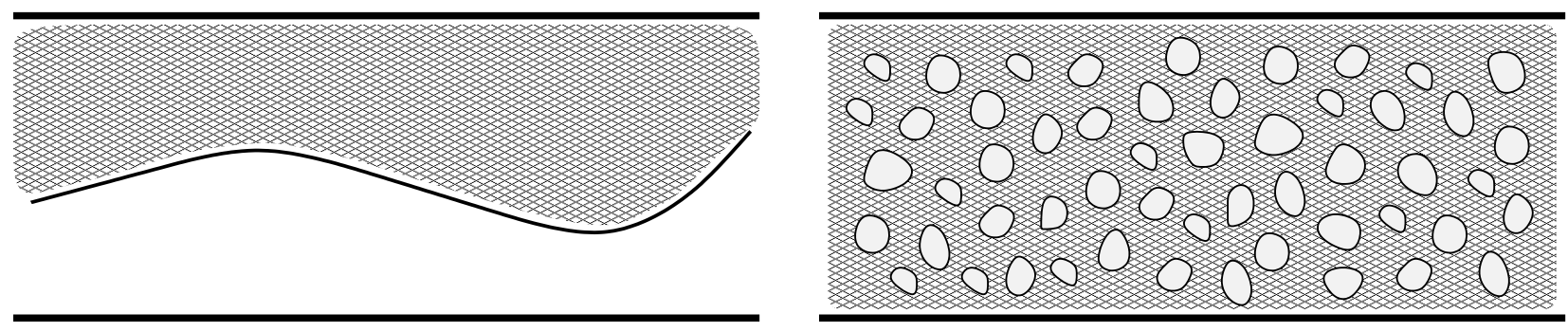

FiguRE 1.1. Separated fluids (left) and dispersed fluid mixture (right).

The inevitable nonconservative form of the average model poses a theoretical difficulty since many problems of interest involve shock waves, whose definition assumes conservation. Generalizations were obtained using products of distributions and traveling wave analysis of vanishing viscosity solutions $[15,19]$. This also poses a computational challenge since numerical discretization approaches rely on the conservation form of the governing equations. Some multiphase models can be formulated as weakly nonconservative, that is as a set of hyperbolic conservation laws with small nonconservative coupling terms. This is the case in two-pressure spray models in which one of the phases is assumed to be an incompressible liquid. The model is only conditionally hyperbolic, provided the volume fraction of the gas phase is close to 1 , and the velocity difference between the phases is small $[15,19]$. Asymptotic shock jump conditions valid for weak shocks were given in [19]. Numerical methods were derived in $[19,22]$. However, these results cannot be extended to general nonconservative models.

The compressible 2-pressure 2-velocity flow model is time-hyperbolic at all flow regimes, but allows for the phases to be out of thermodynamic equilibrium. Restoring equilibrium between the phases via relaxation processes further introduces interphase exchange terms. For example, exchange terms due to velocity nonequilibrium (frictional drag), or due to pressure nonequilibrium.

Other computational challenges include the stiffness due to near incompressibility and fast relaxation terms, preservation of pressure equilibrium among the species and insuring positivity of partial pressures and densities. Several numerical methods have been developed for these models recently. Most relevant for the present paper is the work by Coquel et al. [3] for kinetic schemes and the works by Abgrall and Saurel [2,20] for Godunov-type upwind schemes.

In this paper, we have implemented Godunov-type central and Roe-type upwind schemes to compute compressible two-phase flows. The governing equations are described in Section 2. The central scheme and its implementation is described in Section 3 and the Roe-type upwind scheme is described in Section 4. Numerical examples are presented in Section 5.

\section{THE TWO-PHASE FLOW MODEL}

The flow model is the compressible 2-velocity 2-pressure system following [20]. A closely related model was used in [3]. For notational convenience, we assume a gas phase and a liquid phase, and denote the respective flow variables by the subscript ()$_{g, \ell}$. 
The balance equations are written for the individual phases, which co-exist at every point in space and time with certain volume fractions. An additional equation is written for the volume fraction. The resulting system is

$$
\begin{gathered}
\left(\alpha_{\mathrm{g}}\right)_{t}+U_{\mathrm{i}}\left(\alpha_{\mathrm{g}}\right)_{x}=0 \\
\left(\begin{array}{c}
\alpha_{\mathrm{g}} \rho_{\mathrm{g}} \\
\alpha_{\mathrm{g}} \rho_{\mathrm{g}} u_{\mathrm{g}} \\
\alpha_{\mathrm{g}} E_{\mathrm{g}} \\
\alpha_{\ell} \rho_{\ell} \\
\alpha_{\ell} \rho_{\ell} u_{\ell} \\
\alpha_{\ell} E_{\ell}
\end{array}\right)_{t}+\left(\begin{array}{c}
\alpha_{\mathrm{g}} \rho_{\mathrm{g}} u_{\mathrm{g}} \\
\alpha_{\mathrm{g}} \rho_{\mathrm{g}} u_{\mathrm{g}}^{2}+\alpha_{\mathrm{g}} p_{\mathrm{g}} \\
u_{\mathrm{g}}\left(\alpha_{\mathrm{g}} E_{\mathrm{g}}+\alpha_{\mathrm{g}} p_{\mathrm{g}}\right) \\
\alpha_{\ell} \rho_{\ell} u_{\ell} \\
\alpha_{\ell} \rho_{\ell} u_{\ell}^{2}+\alpha_{\ell} p_{\ell} \\
u_{\ell}\left(\alpha_{\ell} E_{\ell}+\alpha_{\ell} p_{\ell}\right)
\end{array}\right)_{x}=\left(\begin{array}{c}
0 \\
P_{\mathrm{i}}\left(\alpha_{\mathrm{g}}\right)_{x} \\
U_{\mathrm{i}} P_{\mathrm{i}}\left(\alpha_{\mathrm{g}}\right)_{x} \\
0 \\
P_{\mathrm{i}}\left(\alpha_{\ell}\right)_{x} \\
U_{\mathrm{i}} P_{\mathrm{i}}\left(\alpha_{\ell}\right)_{x}
\end{array}\right),
\end{gathered}
$$

where we have used $\rho_{k}, u_{k}$, and $E_{k}$ to denote the density, velocity and energy of the $k$ th phase, and $\alpha_{k}$ to denote its volume fraction, $k \in\{\ell, \mathrm{g}\}$. The total energy of the fluid is given by

$$
E_{k}=\frac{1}{2} \rho_{k} u_{k}^{2}+\rho_{k} e_{k}
$$

and the fluids satisfy the stiffened equation of state

$$
\rho_{k} e_{k}=\frac{p_{k}+\gamma_{k} P_{\infty, k}}{\gamma_{k}-1}
$$

where $e_{k}$ is the specific internal energy of the $k$ th phase, and $P_{\infty, k}$ is a stiffness parameter, with $P_{\infty}=0$ for ideal gases. To close the system, we follow [20] and assume the interface pressure and velocity are given by

$$
\begin{aligned}
P_{\mathrm{i}} & =\alpha_{\mathrm{g}} p_{\mathrm{g}}+\alpha_{\ell} p_{\ell}, \\
U_{\mathrm{i}} & =\frac{\alpha_{\mathrm{g}} \rho_{\mathrm{g}} u_{\mathrm{g}}+\alpha_{\ell} \rho_{\ell} u_{\ell}}{\alpha_{\mathrm{g}} \rho_{\mathrm{g}}+\alpha_{\ell} \rho_{\ell}} .
\end{aligned}
$$

The volume fractions satisfy

$$
\alpha_{\mathrm{g}}+\alpha_{\ell}=1
$$

Enforcing a common pressure on the phases is done by including a pressure relaxation source term. This leaves the hydrodynamic system hyperbolic and circumvents the ill-posedness associated with the single pressure assumption. It is quite possible that with the inclusion of the pressure relaxation terms, the ill-posedness that underlies the system will manifest itself. Velocity relaxation processes (frictional drag) and gravity effects may 
also be included. These effects are summarized in the following source terms:

$$
\vec{S}_{\mathrm{Rel}}=\left(\begin{array}{c}
-\mu\left[p_{\ell}-p_{\mathrm{g}}\right] \\
0 \\
\lambda\left(u_{\ell}-u_{\mathrm{g}}\right) \\
\mu P_{\mathrm{i}}\left[p_{\ell}-p_{\mathrm{g}}\right]+\lambda U_{\mathrm{i}}\left(u_{\ell}-u_{\mathrm{g}}\right) \\
0 \\
-\lambda\left(u_{\ell}-u_{\mathrm{g}}\right) \\
-\mu P_{\mathrm{i}}\left[p_{\ell}-p_{\mathrm{g}}\right]-\lambda U_{\mathrm{i}}\left(u_{\ell}-u_{\mathrm{g}}\right)
\end{array}\right), \quad \vec{S}_{\mathrm{Gr}}=\left(\begin{array}{c}
0 \\
0 \\
\alpha_{\mathrm{g}} \rho_{\mathrm{g}} g \\
\alpha_{\mathrm{g}} \rho_{\mathrm{g}} u_{\mathrm{g}} g \\
0 \\
\alpha_{\ell} \rho_{\ell} g \\
\alpha_{\ell} \rho_{\ell} u_{\ell} g
\end{array}\right),
$$

Here $\mu$ is a pressure relaxation parameter, with $\mu \rightarrow \infty$ corresponding to instantaneous relaxation, $\lambda$ is a velocity relaxation parameter, and $g$ is the gravitational constant.

For later reference, the system (2.1a)-(2.1b) may be written in the form

$$
\begin{aligned}
& \vec{U}_{t}+\vec{F}(\vec{U})_{x}=\vec{S}_{\mathrm{Ex}}+\vec{S}_{\mathrm{Rel}}+\vec{S}_{\mathrm{Gr}}, \quad \text { or } \\
& \vec{U}_{t}+A(\vec{U}) \vec{U}_{x}=\vec{S}_{\mathrm{Rel}}+\vec{S}_{\mathrm{Gr}},
\end{aligned}
$$

where $\vec{F}(\vec{U})$ denotes the (conservative) flux terms, $\vec{S}_{\mathrm{Ex}}$ the (nonconservative) exchange terms between the phases, $A(\vec{U})$ includes both the Jacobian terms, $\frac{\partial \vec{F}}{\partial \vec{U}}$, and the (nonconservative) exchange terms, (and has the eigenstructure (4.1)-(4.3)), and $\vec{S}_{\text {Rel }}$ and $\vec{S}_{\text {Gr }}$ denote the source terms describing relaxation and gravitational effects.

Time integration may be performed by a split-step algorithm, first accounting for the hydrodynamic step (2.1a)-(2.1b), then accounting for relaxation (and other) effects (2.5),

$$
\begin{aligned}
& \text { (i) } \vec{U}_{t}+\vec{F}(\vec{U})_{x}=\vec{S}_{\mathrm{Ex}}+\vec{S}_{\mathrm{Gr}} \text {, or } \quad \text { hydrodynamic step } \\
& \vec{U}_{t}+A(\vec{U}) \vec{U}_{x}=\vec{S}_{\mathrm{Gr}} \text {, } \\
& \text { (ii) } \vec{U}_{t}=\vec{S}_{\mathrm{Rel}} \text { relaxation step }
\end{aligned}
$$

Enforcing a common pressure through relaxation terms leaves the hydrodynamic step (i) time-hyperbolic and, at least technically, circumvents the notorious ill-posedness associated with the 1-pressure model. With the inclusion of the pressure relaxation terms, it is conceivable that the ill-posedness still underlies the system.

In this paper, the hydrodynamic step is realized by the second-order central-upwind scheme and by a Roetype upwind scheme. The schemes are described in Sections 3 and 4, respectively. The relaxation step (ii) follows $[20]$ and is described below for completeness.

\subsection{Pressure relaxation}

In the pressure relaxation step we solve the following system of ODEs,

$$
\begin{aligned}
\left(\alpha_{\mathrm{g}}\right)_{t} & =-\mu\left[p_{\ell}-p_{\mathrm{g}}\right] \\
\left(\alpha_{\mathrm{g}} \rho_{\mathrm{g}}\right)_{t} & =0, \\
\left(\alpha_{\mathrm{g}} \rho_{\mathrm{g}} u_{\mathrm{g}}\right)_{t} & =0, \\
\left(\alpha_{\mathrm{g}} E_{\mathrm{g}}\right)_{t} & =\mu P_{\mathrm{i}}\left[p_{\ell}-p_{\mathrm{g}}\right] .
\end{aligned}
$$


Equations (2.7b) and (2.7c) imply $\left(u_{\mathrm{g}}\right)_{t}=0$, which together with (2.7b) imply

$$
\left(\alpha_{\mathrm{g}} \rho_{\mathrm{g}} u_{\mathrm{g}}^{2}\right)_{t}=0
$$

Equations (2.7a) and (2.7d) give

$$
\left(\alpha_{\mathrm{g}} E_{\mathrm{g}}\right)_{t}=-P_{\mathrm{i}}\left(\alpha_{\mathrm{g}}\right)_{t} .
$$

From $(2.8)$ and the EOS $(2.2 \mathrm{~b})$ the above equation reduces to

$$
\left(\alpha_{\mathrm{g}} \rho_{\mathrm{g}} e_{\mathrm{g}}\right)_{t}=-P_{\mathrm{i}}\left(\alpha_{\mathrm{g}}\right)_{t}
$$

which can be integrated to give

$$
\alpha_{\mathrm{g}} \rho_{\mathrm{g}} e_{\mathrm{g}}=\alpha_{\mathrm{g}}^{o} \rho_{\mathrm{g}}^{o} e_{\mathrm{g}}^{o}-\bar{P}_{\mathrm{i}}\left[\alpha_{\mathrm{g}}-\alpha_{\mathrm{g}}^{o}\right]
$$

where $(\cdot)^{o}=(\cdot)\left(t_{0}\right)$ denotes conditions at the beginning of the relaxation step, and $\bar{P}_{\mathrm{i}}=P_{\mathrm{i}}(\eta)$ is an intermediate value of $P_{\mathrm{i}}$ at $\eta \in\left(t_{0}, t\right)$. Substituting the $\operatorname{EOS}(2.2 \mathrm{~b})$ into $(2.10)$ gives

$$
\alpha_{\mathrm{g}} \frac{p_{\mathrm{g}}+\gamma_{\mathrm{g}} P_{\infty, \mathrm{g}}}{\gamma_{\mathrm{g}}-1}=\alpha_{\mathrm{g}}^{o} \rho_{\mathrm{g}}^{o} e_{\mathrm{g}}^{o}-\bar{P}_{\mathrm{i}}\left(\alpha_{\mathrm{g}}-\alpha_{\mathrm{g}}^{o}\right)
$$

Similarly, for the liquid component we derive

$$
\alpha_{\ell} \frac{p_{\ell}+\gamma_{\ell} P_{\infty, \ell}}{\gamma_{\ell}-1}=\alpha_{\ell}^{o} \rho_{\ell}^{o} e_{\ell}^{o}-\bar{P}_{\mathrm{i}}\left(\alpha_{\ell}-\alpha_{\ell}^{o}\right)
$$

The assumption that the pressure relaxation is infinitely fast $(\mu=\infty)$ implies that the final pressure is common to both phases. We denote by $p:=p_{\mathrm{g}}=p_{\ell}$ the equilibrium pressure, and approximate $\bar{P}_{\mathrm{i}}$ by the midpoint rule $\bar{P}_{\mathrm{i}} \approx \frac{1}{2}\left(p+P_{\mathrm{i}}^{o}\right)$. Eliminating the common pressure $p$ from $(2.11)-(2.12)$, and using $\alpha_{\ell}-\alpha_{\ell}^{o}=-\left(\alpha_{\mathrm{g}}-\alpha_{\mathrm{g}}^{o}\right)$ yields a quadratic equation in $\alpha_{\mathrm{g}}-\alpha_{\mathrm{g}}^{o}$. One root is rejected on physical grounds, the other may be shown to satisfy $\alpha_{\mathrm{g}} \in[0,1]$.

\subsection{Velocity relaxation}

In the velocity relaxation step we solve the following system of ODEs,

$$
\begin{aligned}
\left(\alpha_{\mathrm{g}}\right)_{t} & =0, \\
\left(\alpha_{\mathrm{g}} \rho_{\mathrm{g}}\right)_{t} & =0, \\
\left(\alpha_{\mathrm{g}} \rho_{\mathrm{g}} u_{\mathrm{g}}\right)_{t} & =\lambda\left(u_{\ell}-u_{\mathrm{g}}\right), \\
\left(\alpha_{\mathrm{g}} E_{\mathrm{g}}\right)_{t} & =\lambda U_{\mathrm{i}}\left(u_{\ell}-u_{\mathrm{g}}\right), \\
\left(\alpha_{\ell} \rho_{\ell}\right)_{t} & =0, \\
\left(\alpha_{\ell} \rho_{\ell} u_{\ell}\right)_{t} & =-\lambda\left(u_{\ell}-u_{\mathrm{g}}\right), \\
\left(\alpha_{\ell} E_{\ell}\right)_{t} & =-\lambda U_{\mathrm{i}}\left(u_{\ell}-u_{\mathrm{g}}\right) .
\end{aligned}
$$

First, from (2.13b) and (2.13e) we obtain

$$
\alpha_{\mathrm{g}} \rho_{\mathrm{g}}=\text { Const }, \quad \alpha_{\ell} \rho_{\ell}=\text { Const. }
$$

Then, adding the equations (2.13c) and (2.13f) results in

$$
\alpha_{\mathrm{g}} \rho_{\mathrm{g}}\left(u_{\mathrm{g}}\right)_{t}+\alpha_{\ell} \rho_{\ell}\left(u_{\ell}\right)_{t}=0
$$

which can be integrated to give

$$
\alpha_{\mathrm{g}} \rho_{\mathrm{g}}\left(u_{\mathrm{g}}-u_{\mathrm{g}}^{o}\right)+\alpha_{\ell} \rho_{\ell}\left(u_{\ell}-u_{\ell}^{o}\right)=0
$$


If velocity relaxation is assumed infinitely fast, $(\lambda=\infty), u_{\mathrm{g}}=u_{\ell}=u$ the common velocity, $u$, can be expressed from the above equation,

$$
u=\frac{\alpha_{\mathrm{g}} \rho_{\mathrm{g}} u_{\mathrm{g}}^{o}+\alpha_{\ell} \rho_{\ell} u_{\ell}^{o}}{\alpha_{\mathrm{g}} \rho_{\mathrm{g}}+\alpha_{\ell} \rho_{\ell}} .
$$

The equilibrium velocity is then the velocity of the center of mass [20]. Finite rate velocity relaxation can also be solved in a similar manner.

\section{SECOND-ORDER CENTRAL-UPWIND SCHEME}

Godunov-type central schemes have been advocated as general purpose black-box solvers for hyperbolic conservation laws, and constitute an attractive class of schemes. They avoid the need for solving Riemann problems and are thus simple to implement. Recently, a new class of central schemes, referred to as centralupwind schemes, has been introduced in [9]. They reduce the numerical dissipation, present in staggered central schemes [12], by more careful estimation of the width of the local Riemann fans. They also admit a particularly simple semi-discrete form, and can be readily incorporated in a multistep time integrator. In this paper, a second-order semi-discrete central-upwind scheme has been implemented to solve the two-phase flow system (2.1)-(2.5).

The model consists of six balance laws, (2.1b), and the evolution equation for $\alpha:=\alpha_{g},(2.1 \mathrm{a})$. The system (2.1b) can be written as

$$
\vec{W}_{t}+\vec{F}(\vec{W})_{x}=\vec{G}\left(\vec{W}, \alpha_{x}\right)
$$

with $\vec{G}\left(\vec{W}, \alpha_{x}\right)$ given by

$$
\vec{G}:=\left(0, P_{\mathrm{i}}\left(\alpha_{\mathrm{g}}\right)_{x}+\alpha_{\mathrm{g}} \rho_{\mathrm{g}} g, U_{\mathrm{i}} P_{\mathrm{i}}\left(\alpha_{\mathrm{g}}\right)_{x}+\alpha_{\mathrm{g}} \rho_{\mathrm{g}} u_{\mathrm{g}} g, 0, P_{\mathrm{i}}\left(\alpha_{\ell}\right)_{x}+\alpha_{\ell} \rho_{\ell} g, U_{\mathrm{i}} P_{\mathrm{i}}\left(\alpha_{\ell}\right)_{x}+\alpha_{\ell} \rho_{\ell} u_{\ell} g\right)^{T} .
$$

Gradients of $\alpha$ become difficult to compute accurately if $\alpha$ itself is close to 0 (or 1 ), and errors that are introduced may destabilize the computation. This situation arises, for example, in computing the separation of two phases enclosed in a container. Large $\alpha$ gradients develop near the bottom of the container as the light fluid rises, while its volume fraction becomes very small. In order to stabilize the computation, we follow [3] and define the variable

$$
\beta:=\log \left(\frac{\alpha}{1-\alpha}\right), \quad \text { or } \quad \alpha=\frac{\mathrm{e}^{\beta}}{1+\mathrm{e}^{\beta}} .
$$

Then

$$
\partial \alpha=\alpha(1-\alpha) \partial \beta,
$$

is used in the computations of the derivative of $\alpha$ in (2.1b). This way, inaccuracies in approximating $\partial \beta$ are moderated by the factor $\alpha(1-\alpha)$ which becomes small when $\alpha$ is close to 0 or 1 . Another advantage is that $\alpha$ is guaranteed to be in the range $(0,1)$, and in particular cannot assume negative values even if there are oscillations in $\beta$. This would be more difficult to ensure if $\alpha$ was evolved directly from (2.1a). The Hamilton-Jacobi equation for $\beta$ is the same as the equation for $\alpha$, namely

$$
\beta_{t}+H\left(\beta_{x}\right)=0,
$$

with the Hamiltonian $H(q)=U_{\mathrm{i}} q$.

We compute the solutions of (3.1) and (3.4) using the second-order semi-discrete central-upwind schemes for balance laws [8,9] and Hamilton-Jacobi equations [9]. The semi-discrete central-upwind scheme can be then presented in the following form,

$$
\begin{aligned}
\frac{\mathrm{d} \vec{W}_{j}}{\mathrm{~d} t} & =-\frac{\vec{F}_{j+\frac{1}{2}}-\vec{F}_{j-\frac{1}{2}}}{\Delta x}+\vec{G}\left(\vec{W}_{j},\left(\alpha_{x}\right)_{j}\right), \\
\frac{\mathrm{d} \beta_{j}}{\mathrm{~d} t} & =-H_{j},
\end{aligned}
$$


with the numerical fluxes

$$
\vec{F}_{j+\frac{1}{2}}=\frac{a_{j+\frac{1}{2}}^{+} \vec{F}\left(\vec{W}_{j+\frac{1}{2}}^{-}\right)-a_{j+\frac{1}{2}}^{-} \vec{F}\left(\vec{W}_{j+\frac{1}{2}}^{+}\right)}{a_{j+\frac{1}{2}}^{+}-a_{j+\frac{1}{2}}^{-}}+\frac{a_{j+\frac{1}{2}}^{+} a_{j+\frac{1}{2}}^{-}}{a_{j+\frac{1}{2}}^{+}-a_{j+\frac{1}{2}}^{-}}\left[\vec{W}_{j+\frac{1}{2}}^{+}-\vec{W}_{j+\frac{1}{2}}^{-}\right],
$$

and the numerical Hamiltonian

$$
H_{j}=\left\{\begin{array}{lc}
\left(U_{\mathrm{i}}\right)_{j}\left(\beta_{x}\right)_{j}^{-}, & \text {if }\left(u_{\mathrm{i}}\right)_{j} \geq 0 \\
\left(U_{\mathrm{i}}\right)_{j}\left(\beta_{x}\right)_{j}^{+}, & \text {otherwise. }
\end{array}\right.
$$

The one-sided local speeds of propagation, $a_{j+\frac{1}{2}}^{+}\left(a_{j+\frac{1}{2}}^{-}\right)$, are the positive (negative) part of the largest (smallest) eigenvalues of the Jacobian, $\frac{\partial \vec{F}}{\partial \vec{U}}$, at $x=x_{j+\frac{1}{2}}$, namely

$$
\begin{aligned}
& a_{j+\frac{1}{2}}^{+}=\max _{ \pm}\left\{\max \left\{\left(u_{\mathrm{g}}\right)_{j+\frac{1}{2}}^{ \pm}+\left(c_{\mathrm{g}}\right)_{j+\frac{1}{2}}^{ \pm},\left(u_{\mathrm{g}}\right)_{j+\frac{1}{2}}^{ \pm}-\left(c_{\mathrm{g}}\right)_{j+\frac{1}{2}}^{ \pm},\left(u_{\ell}\right)_{j+\frac{1}{2}}^{ \pm}+\left(c_{\ell}\right)_{j+\frac{1}{2}}^{ \pm},\left(u_{\ell}\right)_{j+\frac{1}{2}}^{ \pm}-\left(c_{\ell}\right)_{j+\frac{1}{2}}^{ \pm}, 0\right\}\right\} \\
& a_{j+\frac{1}{2}}^{-}=\min _{ \pm}\left\{\min \left\{\left(u_{\mathrm{g}}\right)_{j+\frac{1}{2}}^{ \pm}+\left(c_{\mathrm{g}}\right)_{j+\frac{1}{2}}^{ \pm},\left(u_{\mathrm{g}}\right)_{j+\frac{1}{2}}^{ \pm}-\left(c_{\mathrm{g}}\right)_{j+\frac{1}{2}}^{ \pm},\left(u_{\ell}\right)_{j+\frac{1}{2}}^{ \pm}+\left(c_{\ell}\right)_{j+\frac{1}{2}}^{ \pm},\left(u_{\ell}\right)_{j+\frac{1}{2}}^{ \pm}-\left(c_{\ell}\right)_{j+\frac{1}{2}}^{ \pm}, 0\right\}\right\}
\end{aligned}
$$

where the first $\max _{ \pm} / \min _{ \pm}$is taken over the corresponding "+" and "-" values, and the speeds of sound are

$$
\left(c_{k}\right)_{j+\frac{1}{2}}^{ \pm}=\sqrt{\frac{\gamma_{k}\left(\left(p_{k}\right)_{j+\frac{1}{2}}^{ \pm}+P_{\infty, k}\right)}{\left(\rho_{k}\right)_{j+\frac{1}{2}}^{ \pm}}}, \quad k \in\{\ell, \mathrm{g}\}
$$

The values $\vec{W}_{j+\frac{1}{2}}^{ \pm}:=\vec{W}\left(x_{j+\frac{1}{2}} \pm 0\right)$ are reconstructed from the approximate solution $\left\{\vec{W}_{j}\right\}$, and $\left(\beta_{x}\right)_{j}^{ \pm}:=$ $\beta_{x}\left(x_{j} \pm 0\right)$ are the one-sided derivatives of the continuous piecewise parabolic reconstruction for $\beta$, computed at the previous time step.

We implement the reconstruction procedure on the primitive variables $\alpha_{k} \rho_{k}, u_{k}, \alpha_{k} p_{k}$ for $k \in\{\mathrm{g}, \ell\}$, as we have found that this gives better control of the (small) oscillations and tends to reduce them. This does not affect the conservation of $\alpha_{\mathrm{g}} \rho_{\mathrm{g}}, \alpha_{\ell} \rho_{\ell}, \alpha_{\mathrm{g}} \rho_{\mathrm{g}} u_{\mathrm{g}}+\alpha_{\ell} \rho_{\ell} u_{\ell}$ and $\alpha_{\mathrm{g}} E_{\mathrm{g}}+\alpha_{\ell} E_{\ell}$ thanks to the flux form of the semi-discrete scheme (3.5).

\subsection{Piecewise linear reconstructions}

Let $\left\{w_{j}\right\}$ be the cell averages of $w$ ( $w$ is a component of $\left.\vec{W}\right)$. Then the conservative piecewise linear reconstruction has the form,

$$
w_{j}+s_{j}\left(x-x_{j}\right), \quad \text { for } \quad x \in\left[x_{j-\frac{1}{2}}, x_{j+\frac{1}{2}}\right],
$$

with slope $s_{j}$. For second order accuracy, $s_{j}$ needs to be a first-order approximation of $w_{x}\left(x_{j}\right)$. For the scheme to be non-oscillatory, nonlinear limiters are used in the reconstruction.

In this paper, we have used a convex combination of the dissipative minmod $[11,12]$ and the more compressive UNO-like limiter $[6,12]$. The UNO limiter is given by

$$
\mathrm{UNO}_{j}:=\frac{1}{\Delta x} \operatorname{minmod}\left\{\Delta w_{j-\frac{1}{2}}+\frac{1}{2} \operatorname{minmod}\left(\Delta^{2} w_{j-1}, \Delta^{2} w_{j}\right), \Delta w_{j+\frac{1}{2}}-\frac{1}{2} \operatorname{minmod}\left(\Delta^{2} w_{j}, \Delta^{2} w_{j+1}\right)\right\},
$$

where we use the notation,

$$
\Delta w_{j+\frac{1}{2}}:=w_{j+1}-w_{j}, \quad \Delta^{2} w_{j}:=w_{j+1}-2 w_{j}+w_{j-1},
$$


and the multivariate minmod function is defined by

$$
\operatorname{minmod}\left\{x_{1}, x_{2}, \ldots\right\}:=\left\{\begin{array}{lc}
\min \left(x_{1}, x_{2}, \ldots\right), & \text { if } x_{i}>0 \forall i \\
\max \left(x_{1}, x_{2}, \ldots\right), & \text { if } x_{i}<0 \forall i \\
0, & \text { otherwise }
\end{array}\right.
$$

The numerical derivatives, $s_{j}$, are then computed by

$$
s_{j}=\delta \cdot \operatorname{minmod}\left\{\theta \frac{\Delta w_{j-\frac{1}{2}}}{\Delta x}, \frac{\Delta w_{j+\frac{1}{2}}-\Delta w_{j-\frac{1}{2}}}{2 \Delta x}, \theta \frac{\Delta w_{j+\frac{1}{2}}}{\Delta x}\right\}+(1-\delta) \cdot \mathrm{UNO}_{j},
$$

with $\delta \in[0,1]$ and $\theta \in[1,2]$. The parameters $\delta$ and $\theta$ control the balance between the dissipation attributed to the minmod limiter, and the oscillations that may be caused by the UNO-like limiter (see, for example, $[9,10]$ ).

\subsection{Practical implementation of the central-upwind scheme}

In flow situations where the phase volume fractions become small, that is, when the phase "is not there", certain approximation procedures become less accurate, and need to be modified. For example, the computed value of $u_{k}$, obtained through the division of two small numbers $\alpha_{k} \rho_{k} u_{k}$ and $\alpha_{k} \rho_{k}$, may become artificially large, and lead to an unnecessarily severe CFL restriction, due to a phase which is "almost not there". To improve stability and accuracy in these flow situations, we took the following measures.

- The reconstructions of the velocities $u_{k}, k \in\{\mathrm{g}, \ell\}$ always use a more dissipative minmod limiter $((3.10)$ with $\delta=1)$. The reconstruction of the other variables, $\alpha_{k} \rho_{k}$ and $\alpha_{k} p_{k}, k \in\{\mathrm{g}, \ell\}$, and the continuous piecewise parabolic reconstruction of $\beta$ may use a less dissipative limiter $((3.10)$ with $\delta<1)$. The values $\left(\beta_{x}\right)_{j}^{ \pm}$are then used to compute

$$
\left(\beta_{x}\right)_{j}=\frac{\left(\beta_{x}\right)_{j}^{+}+\left(\beta_{x}\right)_{j}^{-}}{2}
$$

needed in (3.3) and (3.5) for discretizing the nonconservative products appearing in $\vec{G}\left(\vec{W}_{j},\left(\alpha_{x}\right)_{j}\right)$. The discretization is performed in a straightforward manner: all terms in $\vec{G}\left(\vec{W}_{j},\left(\alpha_{x}\right)_{j}\right)$ are first obtained from the piecewise polynomial reconstructions, and then their values at $x=x_{j}$ are multiplied.

- In cells $j$ where $\left(\alpha_{\mathrm{g}}\right)_{j}<\varepsilon$ and there is practically no gas, we modify the momentums

$$
\begin{aligned}
\left(\alpha_{\ell} \rho_{\ell} u_{\ell}\right)_{j} & :=\left(\alpha_{\ell} \rho_{\ell} u_{\ell}\right)_{j}+\left(\alpha_{\mathrm{g}} \rho_{\mathrm{g}} u_{\mathrm{g}}\right)_{j} \\
\left(\alpha_{\mathrm{g}} \rho_{\mathrm{g}} u_{\mathrm{g}}\right)_{j}:= & =
\end{aligned}
$$

This modification is conservative, and will automatically give $\left(u_{\mathrm{g}}\right)_{j}=0$ for those cells. The tolerance $\varepsilon$ is a small number, say, $\sim(\Delta x)^{2}$. The case of $\alpha_{\ell} \sim 0$ is treated similarly.

\section{UPWIND SCHEME}

We have implemented a Roe-type upwind scheme $[16,18]$ for the extended system $(2.1)-(2.4)$. The set of dependent variables is $U:=\left(\alpha_{\mathrm{g}}, \alpha_{\mathrm{g}} \rho_{\mathrm{g}}, \alpha_{\mathrm{g}} \rho_{\mathrm{g}} u_{\mathrm{g}}, \alpha_{\mathrm{g}} \rho_{\mathrm{g}} E_{\mathrm{g}}, \alpha_{\ell} \rho_{\ell}, \alpha_{\ell} \rho_{\ell} u_{\ell}, \alpha_{\ell} \rho_{\ell} E_{\ell}\right)^{\mathrm{T}}$. The matrix of right eigenvectors has the recognizable form of the single phase equations with additional entries due to the $\left(\alpha_{\mathrm{g}, \ell}\right)_{x}$ 
exchange terms,

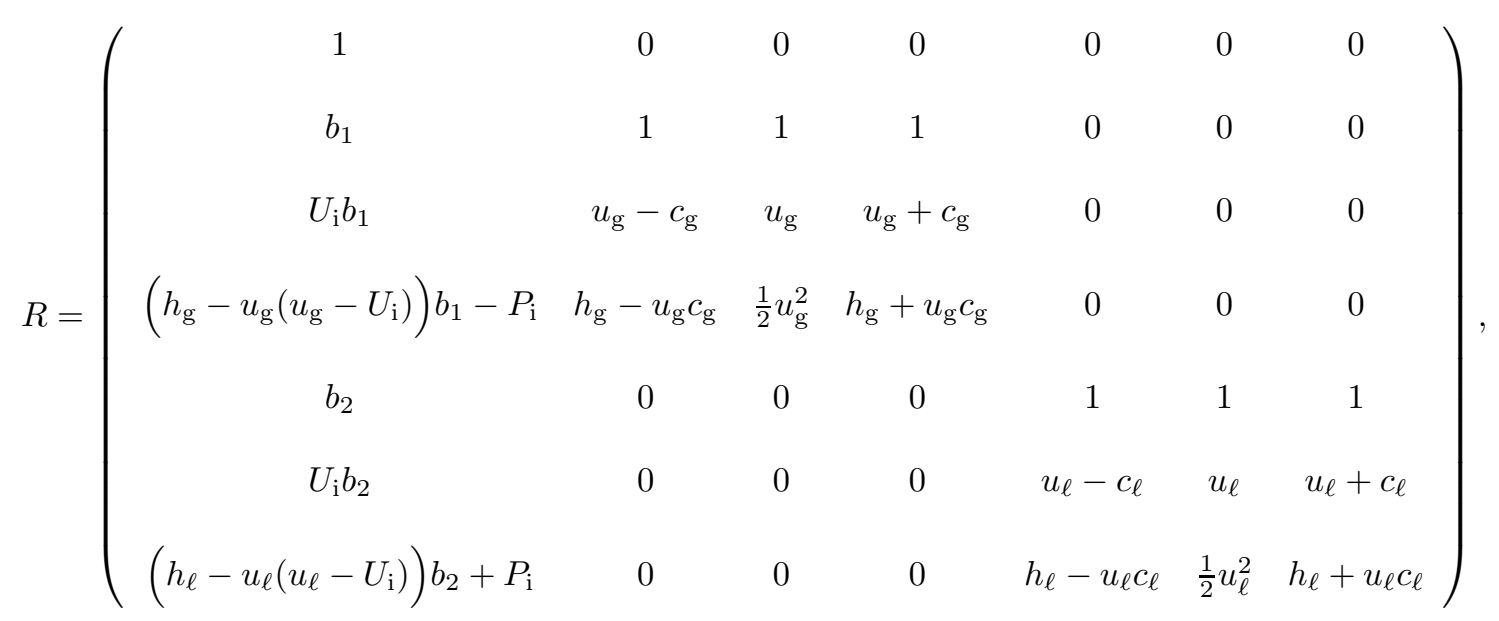

where $h_{k}:=\left(E_{k}+p_{k}\right) / \rho_{k}$ denotes the species specific enthalpy, and

$$
b_{1}=\frac{\gamma_{\mathrm{g}}\left(P_{\mathrm{i}}+P_{\infty, \mathrm{g}}\right)}{c_{\mathrm{g}}^{2}-\left(u_{\mathrm{g}}-U_{\mathrm{i}}\right)^{2}}, \quad b_{2}=-\frac{\gamma_{\ell}\left(P_{\mathrm{i}}+P_{\infty, \ell}\right)}{c_{\ell}^{2}-\left(u_{\ell}-U_{\mathrm{i}}\right)^{2}}
$$

Using $\Delta(\cdot)$ to denote $\Delta(\cdot)=(\cdot)_{R}-(\cdot)_{L}$, the corresponding wave strengths are

$$
\begin{aligned}
\alpha_{1} & =\Delta \alpha_{\mathrm{g}}, \\
\alpha_{2} & =\frac{\Delta\left(\alpha_{\mathrm{g}} p_{\mathrm{g}}\right)-\alpha_{\mathrm{g}} \rho_{\mathrm{g}} c_{\mathrm{g}} \Delta u_{\mathrm{g}}-\left(P_{\mathrm{i}}+\frac{\gamma_{\mathrm{g}}\left(P_{\mathrm{i}}+P_{\infty, \mathrm{g}}\right)}{c_{\mathrm{g}}-\left(u_{\mathrm{g}}-U_{\mathrm{i}}\right)}\left(u_{\mathrm{g}}-U_{\mathrm{i}}\right)\right) \Delta \alpha_{\mathrm{g}}}{2 c_{\mathrm{g}}^{2}}, \\
\alpha_{3} & =\frac{c_{\mathrm{g}}^{2} \Delta\left(\alpha_{\mathrm{g}} \rho_{\mathrm{g}}\right)-\Delta\left(\alpha_{\mathrm{g}} p_{\mathrm{g}}\right)-\left(\left(\gamma_{\mathrm{g}}-1\right) P_{\mathrm{i}}+\gamma_{\mathrm{g}} P_{\infty, \mathrm{g}}\right) \Delta \alpha_{\mathrm{g}}}{c_{\mathrm{g}}^{2}}, \\
\alpha_{4} & =\frac{\Delta\left(\alpha_{\mathrm{g}} p_{\mathrm{g}}\right)+\alpha_{\mathrm{g}} \rho_{\mathrm{g}} c_{\mathrm{g}} \Delta u_{\mathrm{g}}-\left(P_{\mathrm{i}}-\frac{\gamma_{\mathrm{g}}\left(P_{\mathrm{i}}+P_{\infty, \mathrm{g}}\right)}{c_{\mathrm{g}}+\left(u_{\mathrm{g}}-U_{\mathrm{i}}\right)}\left(u_{\mathrm{g}}-U_{\mathrm{i}}\right)\right) \Delta \alpha_{\mathrm{g}}}{2 c_{\mathrm{g}}^{2}} \\
\alpha_{5}= & \frac{\Delta\left(\alpha_{\ell} p_{\ell}\right)-\alpha_{\ell} \rho_{\ell} c_{\ell} \Delta u_{\ell}-\left(P_{\mathrm{i}}+\frac{\gamma_{\ell}\left(P_{\mathrm{i}}+P_{\infty, \ell}\right)}{c_{\ell}-\left(u_{\ell}-U_{\mathrm{i}}\right)}\left(u_{\ell}-U_{\mathrm{i}}\right)\right) \Delta \alpha_{\ell}}{2 c_{\ell}^{2}} \\
\alpha_{6}= & \frac{c_{\ell}^{2} \Delta\left(\alpha_{\ell} \rho_{\ell}\right)-\Delta\left(\alpha_{\ell} p_{\ell}\right)-\left(\left(\gamma_{\ell}-1\right) P_{\mathrm{i}}+\gamma_{\ell} P_{\infty, \ell}\right) \Delta \alpha_{\ell}}{c_{\ell}^{2}}, \\
\alpha_{7}= & \frac{\Delta\left(\alpha_{\ell} p_{\ell}\right)+\alpha_{\ell} \rho_{\ell} c_{\ell} \Delta u_{\ell}-\left(P_{\mathrm{i}}-\frac{\gamma_{\ell}\left(P_{\mathrm{i}}+P_{\infty, \ell}\right)}{c_{\ell}+\left(u_{\ell}-U_{\mathrm{i}}\right)}\left(u_{\ell}-U_{\mathrm{i}}\right)\right) \Delta \alpha_{\ell}}{2 c_{\ell}^{2}}
\end{aligned}
$$

The corresponding eigenvalues are

$$
\Lambda=\left(U_{\mathrm{i}}, u_{\mathrm{g}}-c_{\mathrm{g}}, u_{\mathrm{g}}, u_{\mathrm{g}}+c_{\mathrm{g}}, u_{\ell}-c_{\ell}, u_{\ell}, u_{\ell}+c_{\ell}\right)
$$

and include the familiar single phase speeds, and the interface speed. 
The Roe linearization for this system is

$$
\begin{aligned}
& \overline{\alpha_{k} \rho_{k}}=\sqrt{\left(\alpha_{k} \rho_{k}\right)_{L}\left(\alpha_{k} \rho_{k}\right)_{R}}, \\
& \bar{u}_{k}=\frac{\sqrt{\left(\alpha_{k} \rho_{k}\right)_{L}}\left(u_{k}\right)_{L}+\sqrt{\left(\alpha_{k} \rho_{k}\right)_{R}}\left(u_{k}\right)_{R}}{\sqrt{\left(\alpha_{k} \rho_{k}\right)_{L}}+\sqrt{\left(\alpha_{k} \rho_{k}\right)_{R}}}, \\
& \bar{h}_{k}=\frac{\sqrt{\left(\alpha_{k} \rho_{k}\right)_{L}}\left(h_{k}\right)_{L}+\sqrt{\left(\alpha_{k} \rho_{k}\right)_{R}}\left(h_{k}\right)_{R}}{\sqrt{\left(\alpha_{k} \rho_{k}\right)_{L}}+\sqrt{\left(\alpha_{k} \rho_{k}\right)_{R}}}, \\
& \overline{c_{k}^{2}} \quad=\left(\gamma_{k}-1\right)\left(\bar{h}_{k}-\frac{1}{2} \bar{u}_{k}^{2}\right) .
\end{aligned}
$$

We note that for void wave and contact data,

$$
\begin{aligned}
u_{\mathrm{g}} & =u_{\ell} \quad \Longrightarrow \quad U_{\mathrm{i}}=u_{\mathrm{g}}=u_{\ell} \\
p_{\mathrm{g}} & =p_{\ell} \Longrightarrow P_{\mathrm{i}}=p_{\mathrm{g}}=p_{\ell} \\
\Delta u_{\mathrm{g}} & =0=\Delta u_{\ell} \\
\Delta p_{\mathrm{g}} & =0=\Delta p_{\ell} .
\end{aligned}
$$

A direct calculation shows that the projections onto the acoustic fields are automatically zero, $\alpha_{2}=\alpha_{4}=\alpha_{5}=$ $\alpha_{7}=0$ and that the only nonvanishing projections are onto the void wave and contact wave fields

$$
\alpha_{1}=\Delta \alpha_{\mathrm{g}}, \quad \alpha_{3}=\Delta\left(\alpha_{\mathrm{g}} \rho_{\mathrm{g}}\right)-\rho_{\mathrm{g}} \Delta \alpha_{\mathrm{g}}, \quad \alpha_{6}=\Delta\left(\alpha_{\ell} \rho_{\ell}\right)-\rho_{\ell} \Delta \alpha_{\ell}
$$

A calculation similar to the one in [7] for the single phase Euler system establishes that for this type the pressures and velocities of the phases remain unchanged. This desirable property of preservation of void waves and contact waves is a direct result of including the nonconservative exchange terms in the coefficient matrix of the quasi-linear form (see $\left.(2.6)(i)_{b}\right)$. Splitting the numerical treatment of the flux terms from the exchange terms results in losing this property, and necessitates additional care in the discretization of the volume fraction equation (see for example [20]).

The characteristic field decomposition (4.1)-(4.3) with the local linearization given by (4.4) is implemented in a fluctuation and signal wave propagation scheme [17]. The solution fluctuation $\Delta W$ generates waves (4.1) with strengths (4.2) and speeds (4.3). Each wave is used to update the solution in a Lax-Wendroff type algorithm. The complete solution update is obtained by summing over all the waves. We note that if $\frac{\partial \alpha_{\mathrm{g}}}{\partial x}=0$, the momentum and energy exchange terms vanish and the governing equations decouple into two subsystems in conservation form. In this case, $\Delta \alpha_{\mathrm{g}}=0$ and the characteristic wave decomposition (4.1) $-(4.3)$ reduces to the familiar eigenstructure of the Euler system. The Roe-type linearization (4.4) ensures that the algorithm is then conservative, even though an explicit flux function is not formulated. The interface pressure and velocity are given by (2.3).

\section{Numerical EXAMPleS}

We first make a general comment on positivity aspects of the computed solutions. If $\alpha_{k} \sim 0$, the partial density $\alpha_{k} \rho_{k}$ is small and even a small numerical oscillation may cause it to become negative. This in turn may cause the computation to break down. For these flow regimes, a desirable scheme property is positivity preservation, a property that ensures that if the partial densities are positive in the data, they remain so in the numerical solution. 
The semi-discrete central-upwind scheme (3.5)-(3.8) forms a set of ODEs which is integrated by the secondorder modified Euler method. It can be shown that by restricting the CFL number to 0.25 (instead of the usual 0.5), the partial densities remain positive (the proof is completely analogous to the proof of Theorem 4.1 in [8]). This restriction may unnecessarily penalize the computation. To prevent this, we use the more restrictive timestep only when needed. We have implemented the following time integration strategy: we evolve the solution from time $t$ to time $t+\Delta t$ using the CFL number of 0.5. If any of the computed values of $\alpha_{k} \rho_{k}$ is negative, this evolution step is rejected, and instead the solution is evolved from time $t$ to $t+\Delta t / 2$. Our numerical experiments indicate that only a few evolution steps are rejected.

The upwind scheme described in Section 4 is of Roe type [17]. Similar to the single phase Roe solver, the method is not guaranteed to maintain positive densities [5]. Depending on the problem, the method may or may not fail near very low partial densities. We comment further on this issue in the numerical examples below.

Example 1. - Propagation of void waves

Figure 5.1 shows the propagation of a void wave by the upwind scheme (4.1)-(4.4). The initial data is given by

$$
\begin{array}{ll}
u_{\mathrm{g}}=u_{\ell}=1, & p_{\mathrm{g}}=p_{\ell}=1, \\
\gamma_{\mathrm{g}}=1.4, & \gamma_{\ell}=1.2, \\
\left(\rho_{\mathrm{g}}\right)_{L}=2, & \left(\rho_{\mathrm{g}}\right)_{R}=1, \\
\left(\rho_{\ell}\right)_{L}=1, & \left(\rho_{\ell}\right)_{R}=2, \\
\left(\alpha_{\mathrm{g}}\right)_{L}=0.1, & \left(\alpha_{\mathrm{g}}\right)_{R}=0.9 .
\end{array}
$$

This data corresponds to a void wave which is also a contact surface in each of the phases. This wave separates two phases that are in mechanical equilibrium. The results confirm that mechanical equilibrium between the phases is preserved, with pressures and velocities remaining uniform.

\section{Example 2. - The multifluid limit}

In this example, we test the multiphase flow solver near the multifluid limit of two pure fluids separated by an interface. In the multiphase flow model, this corresponds to a region occupied by only one phase, where $\alpha \sim 1$, and a region occupied only by the other phase, where $\alpha \sim 0$. The multifluid limit assumes that the different phases may be described by a single velocity and a single pressure function. This enables the equations of motion to be written for the fluid mixture, and exact solutions are available in one space dimension. Recently, this reduced system has attracted much interest and has been the focus of design of many successful numerical schemes (see [1] and references cited therein).

The multiphase flow model is more general, and allows the respective phases to possess their own velocities and pressures. The multifluid limit is implemented by including pressure relaxation and velocity relaxation terms (2.5), with instantaneous relaxation rates $(\lambda=\mu=\infty)$.

The first example corresponds to an air-helium shock-tube data

$$
\begin{array}{lrl} 
& \left(\rho_{\mathrm{g}}, u_{\mathrm{g}}, p_{\mathrm{g}}, \gamma_{\mathrm{g}}, P_{\infty, \mathrm{g}}\right)= & (4 / 29,0,0.1,5 / 3,0), \\
\text { helium : } & \left(\rho_{\ell}, u_{\ell}, p_{\ell}, \gamma_{\ell}, P_{\infty, \ell}\right)= & (1,0,1,1.4,0), \\
\text { air : } & \left(\alpha_{\mathrm{g}}\right)_{\mathrm{L}}=10^{-8} \sim 0, & \left(\alpha_{\mathrm{g}}\right)_{\mathrm{R}}=1-10^{-8} \sim 1 . \\
\text { volume fraction: } &
\end{array}
$$

Computed results by the second-order upwind scheme with the minmod limiter on a 400 point mesh, together with the exact solution, are shown in Figure 5.2 and are in excellent agreement. We note here that although the upwind scheme is not guaranteed to maintain positive partial densities, the partial densities, while extremely small, remain positive.

The next example corresponds to a water-air shock-tube data

$$
\begin{array}{lll}
\text { air : } & \left(\rho_{\mathrm{g}}, u_{\mathrm{g}}, p_{\mathrm{g}}, \gamma_{\mathrm{g}}, P_{\infty, \mathrm{g}}\right)= & \left(65.0,0.0,10^{5}, 1.4,0.0\right), \\
\text { water : } & \left(\rho_{\ell}, u_{\ell}, p_{\ell}, \gamma_{\ell}, P_{\infty, \ell}\right)= & \left(1000.0,0.0,10^{9}, 4.4,6 \cdot 10^{8}\right), \\
\text { volume fraction: } & \left(\alpha_{\mathrm{g}}\right)_{\mathrm{L}}=10^{-8} \sim 0, & \left(\alpha_{\mathrm{g}}\right)_{\mathrm{R}}=1-10^{-8} \sim 1 .
\end{array}
$$



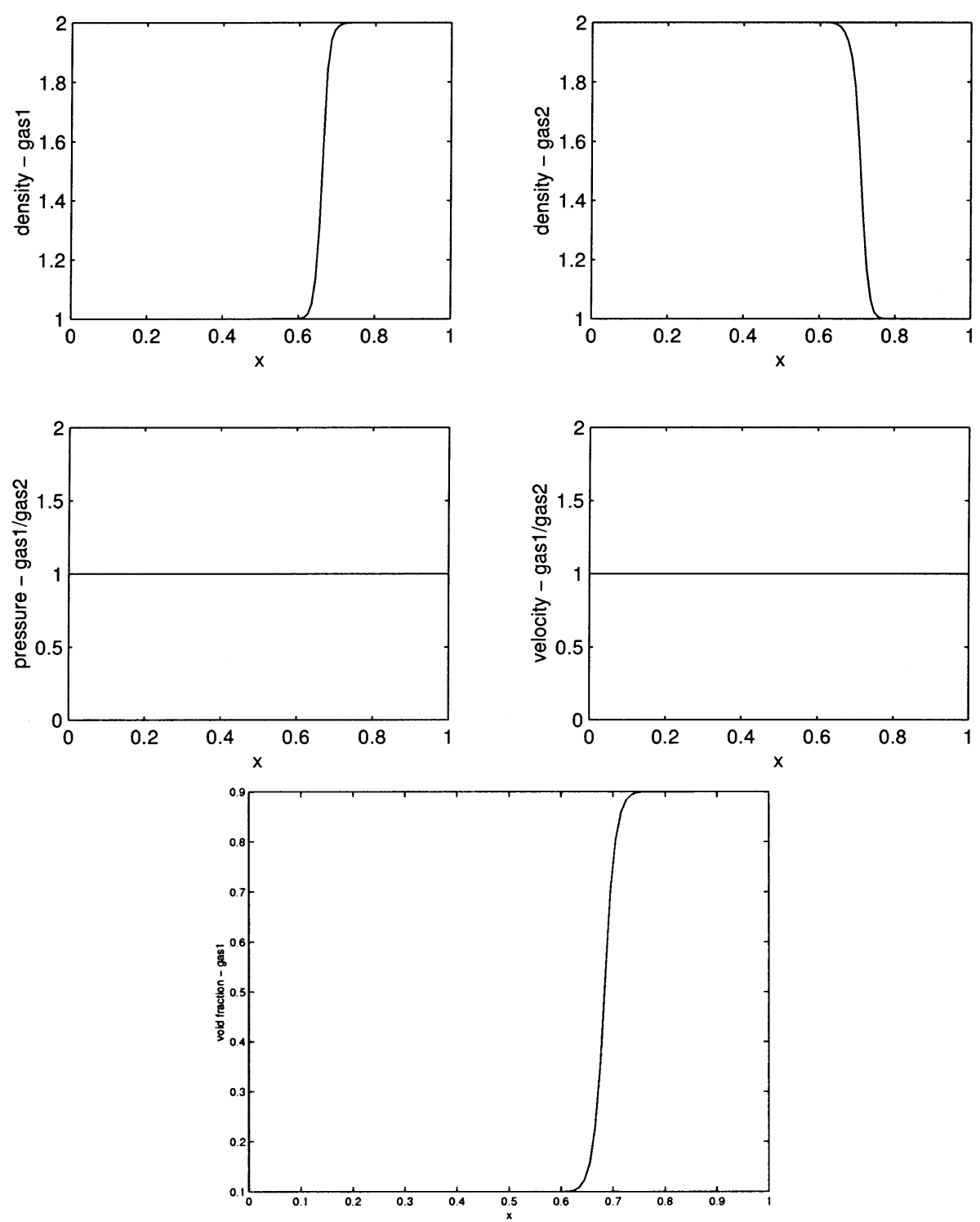

FiguRE 5.1. Propagation of a void wave.

Computed results by the second-order upwind scheme on a 400 point mesh with the minmod limiter, together with the exact solution, are shown in Figure 5.3 and are again in excellent agreement. Again the computed partial densities, while extremely small, remains positive.

\section{Example 3. - Gas-gas sedimentation problem}

In this example, we consider the ideal gas mixture of air $(\ell)$ and helium $(\mathrm{g})$ enclosed in a container of length 1. We take $\gamma_{\mathrm{g}}=\frac{5}{3}, \gamma_{\ell}=1.4$, and $P_{\infty, \mathrm{g}}=P_{\infty, \ell}=0$. The nondimensionalized gravity constant is $g=\frac{1}{30}$, and the initial data are

$$
\rho_{\mathrm{g}}=\frac{4}{29}, \quad \rho_{\ell}=1, \quad u_{\mathrm{g}}=u_{\ell}=0, \quad p_{\mathrm{g}}=p_{\ell}=1, \quad \alpha_{\mathrm{g}}=\alpha_{\ell}=0.5
$$



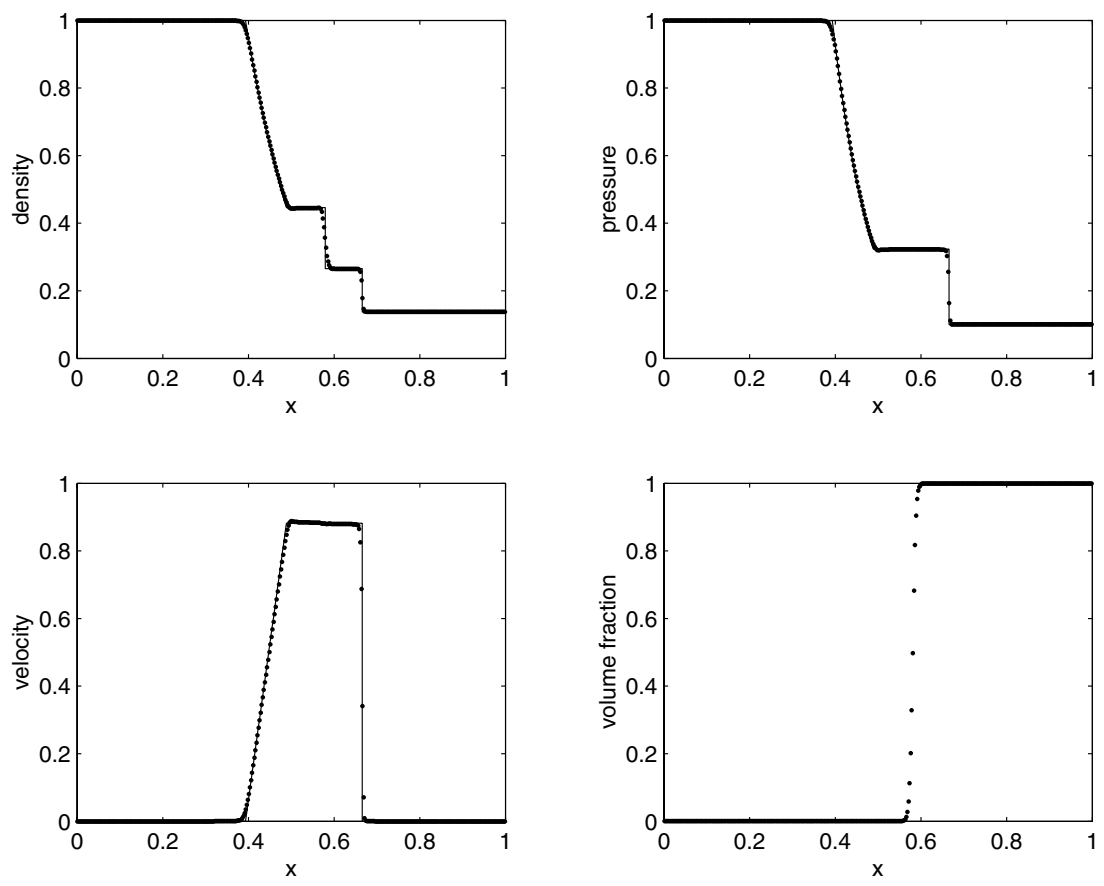

FIGURE 5.2. Multifluid limit: air-helium shock-tube problem.
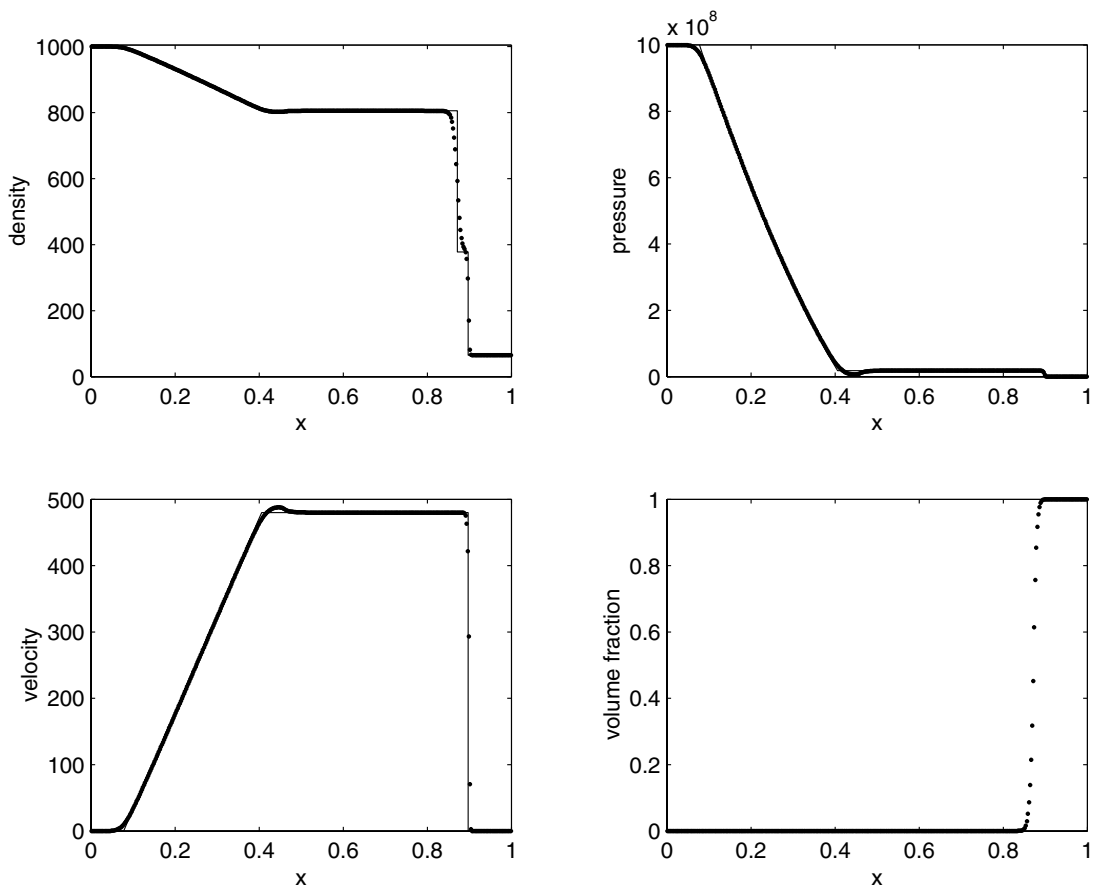

FIGURE 5.3. Multifluid limit: water-air shock-tube problem. 
Air is heavier than helium. As a result the air slowly moves down (to the right in our setting) and the helium moves up (left) due to gravity, until they separate. Solid wall boundary conditions are imposed on both ends of the domain. The solution is computed using 200 grid points, and the minmod limiter, (3.10) with $\delta=1$

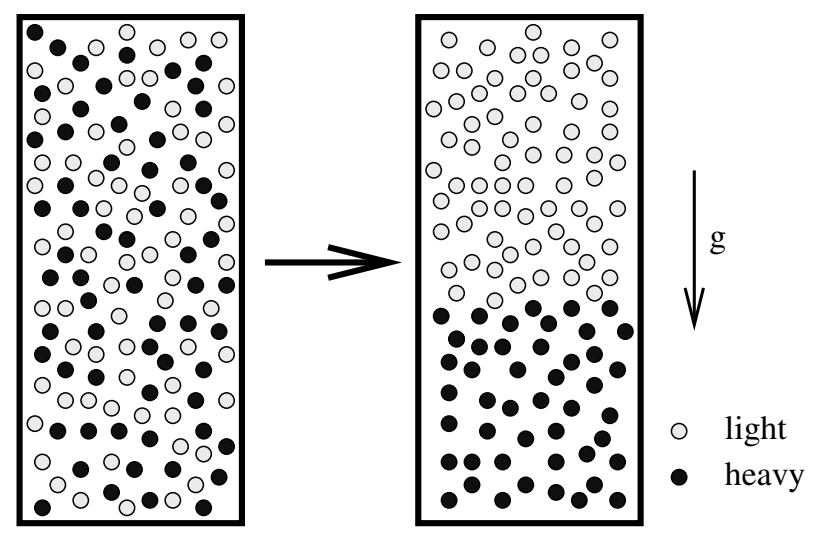

FIGURE 5.4. Separation of two fluids in a gravitational field.

and $\theta=1.3$. The volume fraction of both gases is set initially to $\alpha_{k}=0.5$. The time evolution of the volume fraction of the helium $\alpha_{\mathrm{g}}$ is shown in Figure 5.5a. During the sedimentation process, the helium rises from the bottom and accumulates at the top, causing $\alpha_{\mathrm{g}}$ to decrease near the bottom (right) boundary and increase near the top (left) boundary. At time $T=10$ the fluids are essentially separated, with $\alpha_{\mathrm{g}} \sim 0$ near the bottom and $\alpha_{\mathrm{g}} \sim 1$ near the top. The evolution of the total density of the mixture is presented in Figure 5.5b.

Example 4. - Water-air sedimentation problem

The next example consists of a tube of length 1, initially filled with a homogeneous two-phase mixture of water $\left(\gamma_{\ell}=4.4, P_{\infty, \ell}=6 \times 10^{8}\right)$ and air $\left(\gamma_{\mathrm{g}}=1.4, P_{\infty, \mathrm{g}}=0\right)$. The initial data is

$$
\rho_{\mathrm{g}}=1, \quad \rho_{\ell}=1000, \quad u_{\mathrm{g}}=u_{\ell}=0, \quad p_{\mathrm{g}}=p_{\ell}=10^{5}, \quad \alpha_{\mathrm{g}}=\alpha_{\ell}=0.5 .
$$

Solid wall boundary conditions are imposed on both ends of the tube. The phases separate due to gravity $(g=9.8)$ : the water moves down (to the right), while the air moves up (to the left).

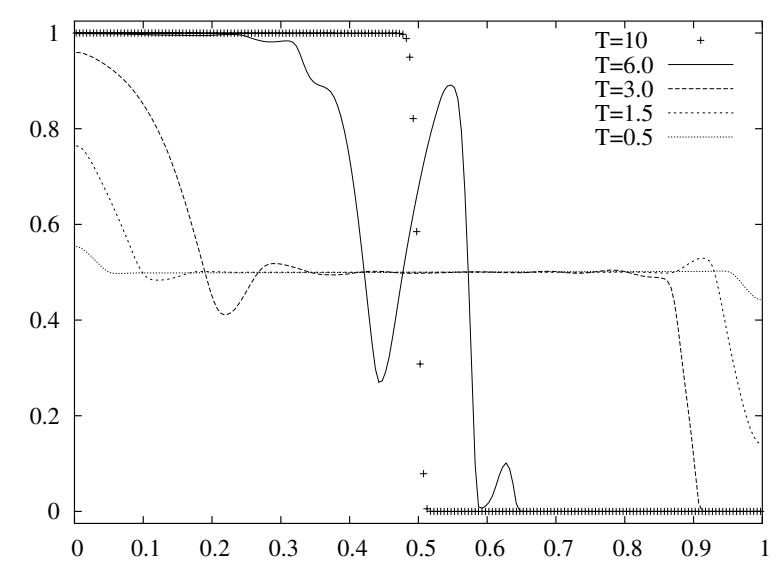

Figure 5.5A. Helium volume fraction, $\alpha_{\mathrm{g}}$.

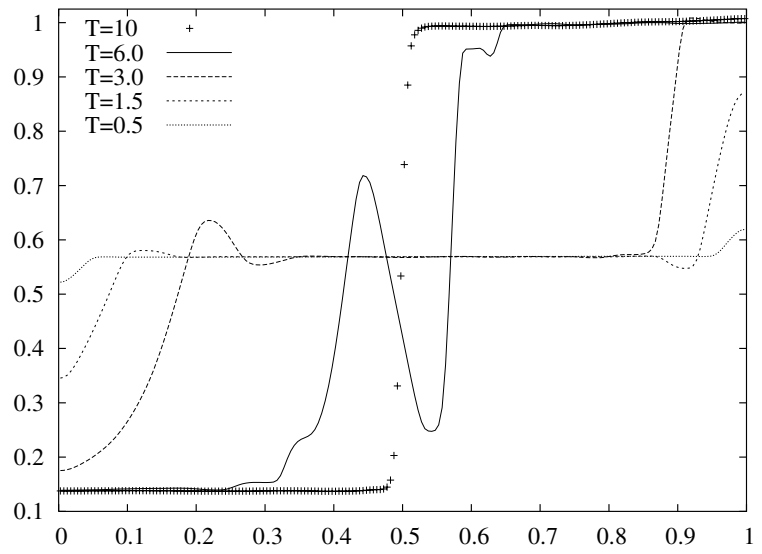

Figure 5.5B. Total density, $\alpha_{\mathrm{g}} \rho_{\mathrm{g}}+\alpha_{\ell} \rho_{\ell}$. 


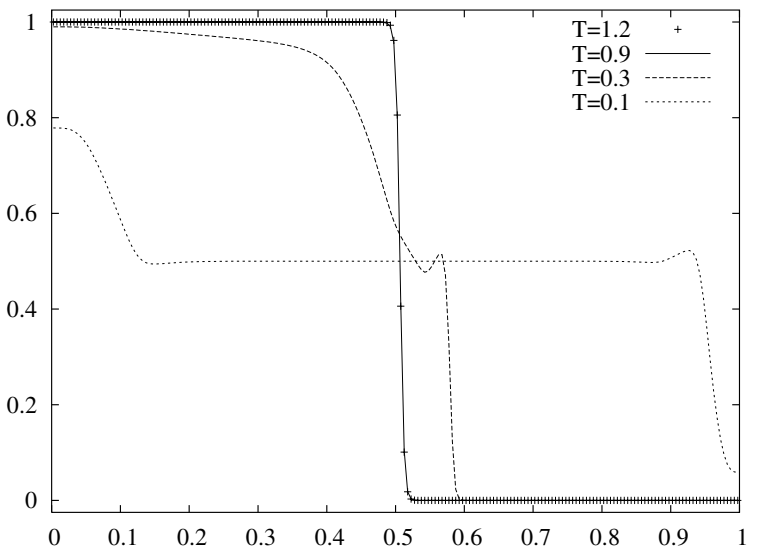

Figure 5.6A. Air volume fraction, $\alpha_{\mathrm{g}}$.

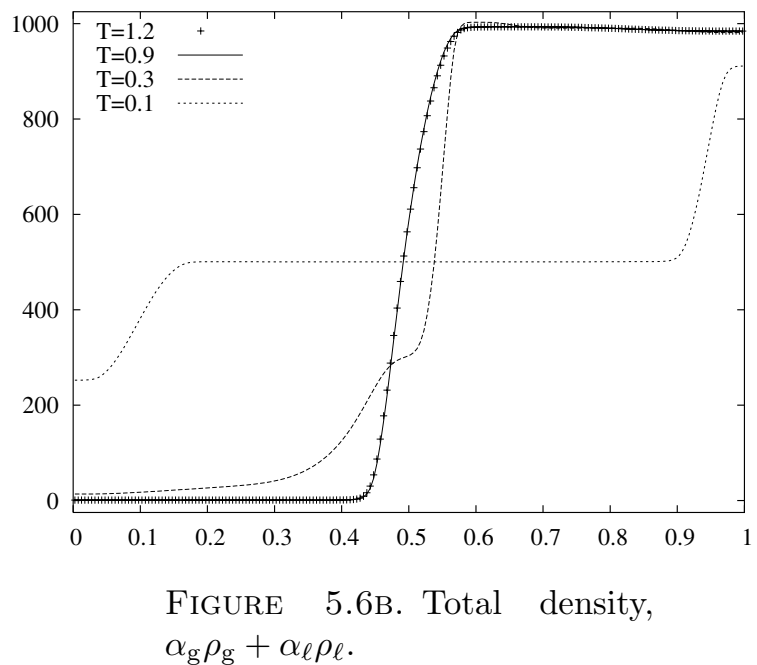

$\alpha_{\mathrm{g}} \rho_{\mathrm{g}}+\alpha_{\ell} \rho_{\ell}$.

The solution is computed using 200 grid points, and the minmod reconstruction, (3.10) with $\delta=1$ and $\theta=1.3$. We show the separation of the two phases by plotting the volume fraction of the air - Figure $5.6 \mathrm{a}$, and the total density of the mixture - Figure 5.6b.

We note that the interface between the two phases at large times $(T=0.9,1.2)$ is very well resolved in Figure $5.6 \mathrm{a}$, while it is much more smeared in Figure 5.6b. This happens because, in fact, we evolve $\alpha_{\mathrm{g}}$ separately from the other variables, and this allows us to use more accurately estimated local speeds of propagation for the volume fraction, as described in Section 3. However, our numerical experiments (not shown in this paper) indicate convergence in all fields as the mesh is refined.

\section{Example 5. - Water faucet problem}

In this example, we test our numerical methods on the water faucet problem, proposed in [13], see also [3,20]. Water flows at constant rate into the top of a vertical tube of length $12 \mathrm{~m}$. The tube is open to ambient pressure at the bottom.

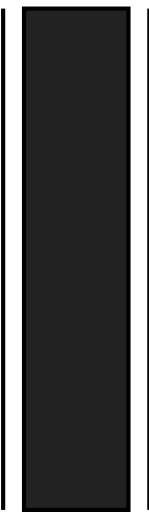

(a)

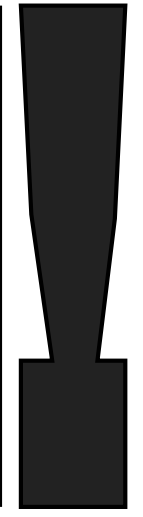

(b)

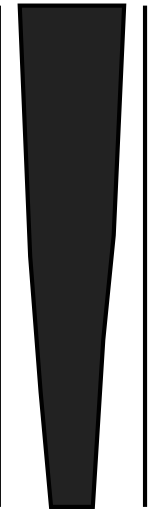

(c)

FiguRE 5.7. Water faucet problem (a) $t=0$, (b) $t>0$, and (c) $t \gg 0$.

The initial data is $\rho_{\mathrm{g}}=1, \rho_{\ell}=1000, u_{\mathrm{g}}=0, u_{\ell}=10, p_{\mathrm{g}}=p_{\ell}=10^{5}, \alpha_{\mathrm{g}}=0.2, \alpha_{\ell}=0.8$, with inlet boundary conditions: $u_{\mathrm{g}}=0, u_{\ell}=10, \alpha_{\mathrm{g}}=0.2$, and outlet boundary conditions: $p_{\mathrm{g}}=p_{\ell}=10^{5}$. 


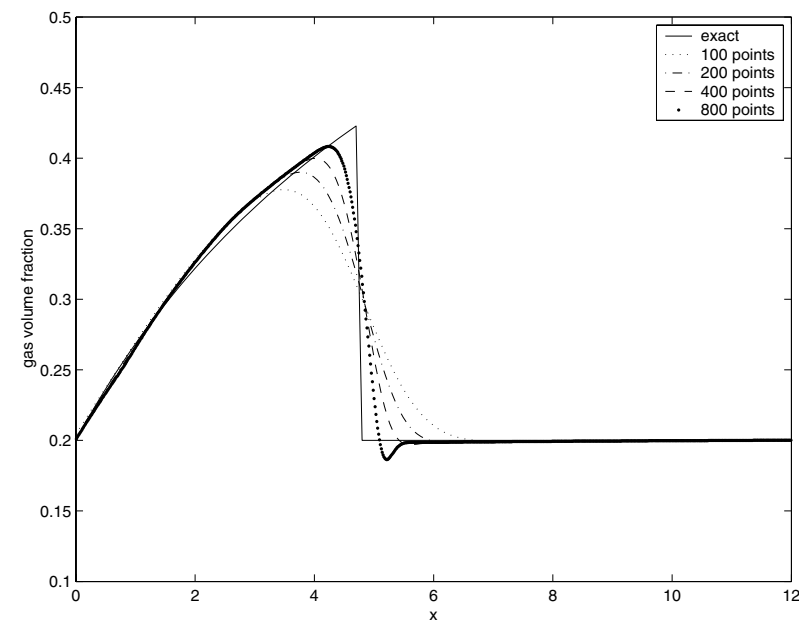

FIGURE 5.8A. $\alpha_{\mathrm{g}}$ by 1st-order upwind scheme.

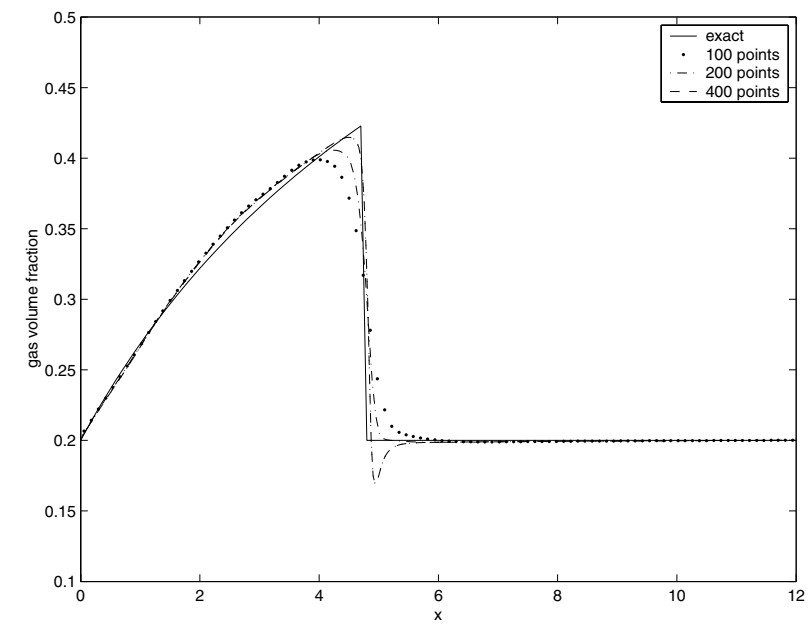

FiguRE 5.8B. $\alpha_{\mathrm{g}}$ by 2nd-order upwind scheme.

If pressure variation is ignored, this problem has an explicit solution (see $[3,13,20])$,

$$
\alpha_{\mathrm{g}}(x, t)=\left\{\begin{array}{lc}
1-\frac{8}{\sqrt{100+19.6 x}}, & \text { for } x<10 t+4.9 t^{2}, \\
0.2, & \text { otherwise. }
\end{array}\right.
$$

The solution is computed at time $T=0.4$, using a sequence of grids, $N=100,200,400$, and 800 .

In this example, the water is modeled by an ideal gas with $\gamma_{\ell}=1.0005$ following [3]. The gravitational constant is $g=9.8$. Figures $5.8 \mathrm{a}$ and $5.8 \mathrm{~b}$ show the numerical results obtained by the first- and secondorder upwind scheme. They are in very good agreement with the exact solution. We note in both figures the appearance of a small undershot, which becomes more noticeable in the second-order computation. This undershot may be a manifestation of the underlying ill-posedness of the 1-pressure 2-velocity two-phase flow model.

We have also modeled the water by the stiff EOS with $\gamma_{\ell}=4.4$ and $P_{\infty, \ell}=6 \cdot 10^{8}$. Figures $5.9 \mathrm{a}$ and $5.9 \mathrm{~b}$ show the volume fraction $\alpha_{\mathrm{g}}$, computed by the second-order central-upwind scheme using the limiter (3.10) with $\delta=0$ and $\delta=0.2$. Agreement with the exact solution is very good. As can be observed, the more compressive limiter (a smaller value of $\delta$ ) produces results with better resolution, but also a larger over- and undershootings near the shock discontinuity. While the oscillation may be attributed to the reduced dissipation in Figure 5.9a, it may again be a manifestation of the underlying ill-posedness of the 1-pressure 2-velocities two-phase flow model.

Acknowledgements. The work of S. Karni was supported in part by NSF Grant \# DMS-9973291, and by ONR Award \# N00014-99-1-0449. S. Karni wishes to acknowledge with gratitude the hospitality of Prof. Vered Rom-Kedar of the Weizmann Institute of Science, Israel. E. Kirr was supported in part by ONR Award \# N00014-99-1-0449. The work of A. Kurganov was supported in part by the NSF Group Infrastructure Grant and the NSF Grants \# DMS-0196439, DMS-0310585. G. Petrova was supported in part by NSF Grant \# DMS-0296020. 


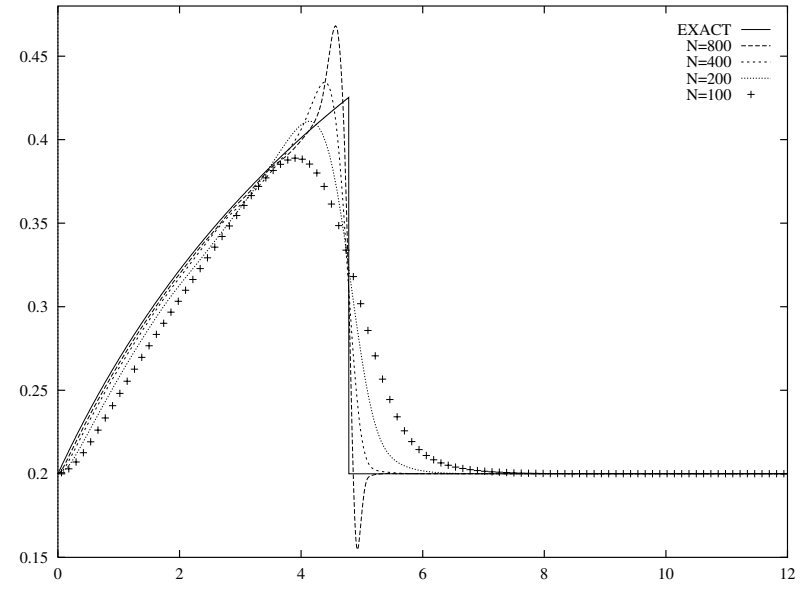

FiguRE 5.9A. $\alpha_{\mathrm{g}}$ with $\delta=0$.

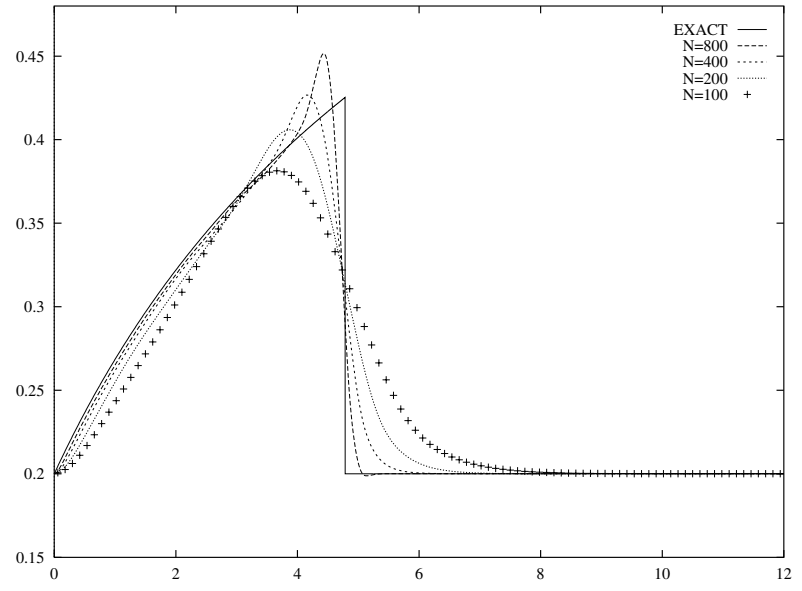

FiguRE 5.9B. $\alpha_{\mathrm{g}}$ with $\delta=0.2$.

\section{REFERENCES}

[1] R. Abgrall and S. Karni, Computations of compressible multifluids. J. Comput. Phys. 169 (2001) 594-623.

[2] R. Abgrall and R. Saurel, Discrete equations for physical and numerical compressible multiphase flow mixtures. J. Comput. Phys. 186 (2003) 361-396.

[3] F. Coquel, K. El Amine, E. Godlewski, B. Perthame and P. Rascle, A numerical method using upwind schemes for the resolution of two-phase flows. J. Comput. Phys. 136 (1997) 272-288.

[4] D.A. Drew, Mathematical modelling of tow-phase flow. Ann. Rev. Fluid Mech. 15 (1983) 261-291.

[5] B. Einfeldt, C.-D. Munz, P.L. Roe and B. Sjogreen, On Godunov-type methods near low densities. J. Comput. Phys. 92 (1991) 273-295.

[6] A. Harten and S. Osher, Uniformly high-order accurate nonoscillatory schemes. I. SIAM J. Numer. Anal. 24 (1987) $279-309$.

[7] S. Karni, Multi-component flow calculations by a consistent primitive algorithm. J. Comput. Phys. 112 (1994) 31-43.

[8] A. Kurganov and D. Levy, Central-upwind schemes for the Saint-Venant system. ESAIM: M2AN 36 (2002) $397-425$.

[9] A. Kurganov, S. Noelle and G. Petrova, Semi-discrete central-upwind schemes for hyperbolic conservation laws and HamiltonJacobi equations. SIAM J. Sci. Comput. 23 (2001) 707-740.

[10] A. Kurganov and G. Petrova, Central schemes and contact discontinuities. ESAIM: M2AN 34 (2000) 1259-1275.

[11] B. van Leer, Towards the ultimate conservative difference scheme, V. A second order sequel to Godunov's method. J. Comput. Phys. 32 (1979) 101-136.

[12] H. Nessyahu and E. Tadmor, Non-oscillatory central differencing for hyperbolic conservation laws. J. Comput. Phys. 87 (1990) 408-463.

[13] V.H. Ransom, Numerical benchmark tests, G.F. Hewitt, J.M. Delhay and N. Zuber Eds., Hemisphere, Washington, DC Multiphase Science and Technology 3 (1987).

[14] P.-A. Raviart and L. Sainsaulieu, Nonconservative hyperbolic systems and two-phase flows, International Conference on Differential Equations (Barcelona, 1991) World Sci. Publishing, River Edge, NJ 1, 2 (1993) 225-233.

[15] P.-A. Raviart and L. Sainsaulieu, A nonconservative hyperbolic system modeling spray dynamics. I. Solution of the Riemann problem. Math. Models Methods Appl. Sci. 5 (1995) 297-333.

[16] P.L. Roe, Approximate Riemann solvers, parameter vectors and difference schemes. J. Comput. Phys. 43 (1981) $357-372$.

[17] P.L. Roe, Fluctuations and signals - A framework for numerical evolution problems, in Numerical Methods for Fluid Dynamics, K.W. Morton and M.J. Baines Eds., Academic Press (1982) 219-257.

[18] P.L. Roe and J. Pike, Efficient construction and utilisation of approximate Riemann solutions, in Computing methods in applied sciences and engineering, VI (Versailles, 1983) North-Holland, Amsterdam (1984) 499-518.

[19] L. Sainsaulieu, Finite volume approximations of two-phase fluid flows based on an approximate Roe-type Riemann solver. J. Comput. Phys. 121 (1995) 1-28.

[20] R. Saurel and R. Abgrall, A multiphase Godunov method for compressible multifluid and multiphase flows. J. Comput. Phys. 150 (1999) 425-467.

[21] H.B. Stewart and B. Wendroff, Two-phase flow: models and methods. J. Comput. Phys. 56 (1984) 363-409.

[22] I. Toumi and A. Kumbaro, An approximate linearized Riemann solver for a two-fluid model. J. Comput. Phys. 124 (1996) 286-300. 